\title{
'Intergenerational Gap' Might Play a Stronger Role than Socio-Cultural Gap in Contemporary Age Perception
}

\author{
Ha Na Song, Soyoung Park and Eung Kwon Pae* \\ Department of Orthodontics and Pediatric Dentistry, School of Dentistry, University of Maryland, Baltimore, Maryland, USA
}

*Corresponding author: Eung Kwon Pae, Department of Orthodontics and Pediatric Dentistry, School of Dentistry, University of Maryland, 650 W, Baltimore Street, Baltimore, MD 21201, USA, Tel: 310-740-6161; E-mail: eungkpae@gmail.com

Received: 22 Mar, 2019 | Accepted: 28 Mar, 2019 | Published: 04 Apr, 2019

Citation: Song HN, Park S, Pae EK (2019) 'Intergenerational Gap' Might Play a Stronger Role than Socio-Cultural Gap in Contemporary Age Perception. Int J Dent Oral Health 5(2): dx.doi.org/10.16966/2378-7090.290

Copyright: (C) 2019 Song HN, et al. This is an open-access article distributed under the terms of the Creative Commons Attribution License, which permits unrestricted use, distribution, and reproduction in any medium, provided the original author and source are credited.

\section{Abstract}

Introduction: This study aimed to examine differences in inter-sociocultural perspectives among orthodontists assessing post-surgical facial appearance changes of Korean patients with 'guessing age' of the patients as a tool.

Methods: 20 orthodontists practicing in US and Korea examined facial photographs of 14 patients ( 7 male and 7 female) with skeletal Class III who underwent surgery-first orthognathic correction, facial bone contouring and which followed by post-surgical orthodontic treatments by an oral surgeon, a plastic surgeon and an orthodontist respectively. Two groups (US and Korea) of examiners postulated patients' perceiving age after observing frontal repose, frontal smile, 45 degrees and profile views of final (T2) first then initial (T1) photographs of each patient in such order. The data was analyzed according to examiner's practicing years (younger vs older group) and nationality (US vs Korea). Generalized Linear Model was used to compensate random effects for categorical variable.

Results: Unexpectedly, a significant generation-gap emerged in both nationalities. Both older groups in US and Korea perceived less age difference (i.e., less improvement from the surgery) between $\mathrm{T} 2$ and $\mathrm{T} 1$ than the younger groups. In comparison with male patients, the age estimation of female patients revealed stronger generation-gap (i.e., lesser differences between $\mathrm{T} 2$ and $\mathrm{T} 1$ ).

Conclusions: Our results showed, to certain extent, a cross-cultural consensus on the effects of the combination surgery is advantageous to enhance perception of 'youthfulness' in the face. Evidently, modern-day standard of beauty has become global; however, considering the different results from the U.S. older groups, generation-gap perhaps plays a more significant role in social cognition.

Keywords: Age perception; Intergenerational gap; Orthognathic surgery

\section{Introduction}

We decide, "Almost instantaneously what we like and the decisions are highly consistent in our assessments, even across cultures" [1]. Differences in perception on facial beauty and attractiveness were often studied based on socio-economies or ethnicities [2-6]; however, no study has revealed an inter-generational gap in experts' opinions on perceptional age. A significant agreement between White Scottish and Black South African observers' attractiveness judgments was reported [7]. This provides an evidence of strong cross-cultural agreement in facial attractiveness preferences. A new question arises, then, whether an intergeneration gap exists in opinions on perception in such a rapid changing modern culture. This question is important because opinions on youthfulness and attractiveness of human faces are considered universal due to a spatial and temporal connectivity among global regions and cultures via IT technologies forcing the world toward asymptotic communality. Most previous studies investigated disparities in public opinions based on nationality, socio- economic status, ethnic and cultural differences. However, differential opinions upon such various backgrounds may be becoming less significant than an intergenerational gap because a rapid dynamic cultural current in facial perception could override rather static geographical cultural variances in different populations. If an intergenerational 'acculturation-gap' does exist among orthodontists, we wanted to test the conjecture that 'digital acculturation' surpasses physical enculturation in rapid changing modern orthodontic society. We want to be perceived attractive and attractiveness is associated with youthfulness to some degree. Yet, a recent work reported that higher attractiveness ratings, together with older aged faces, led to more positive evaluations of competence [8]. Therefore, orthodontists need to develop keen opinions and knowledge on this social issue of relationship between facial attractiveness and age perception. Purpose of this preliminary study was to examine differences in intergenerational and inter-sociocultural perspectives among orthodontists assessing post-surgical facial appearance change of Asian patients with an emphasis on estimating age of the patients. 


\section{Subjects and Methods}

Twenty orthodontists table 1 practicing in Korea $(n=10$ including 3 female and 7 male orthodontists) and US ( $\mathrm{n}=10$ including 4 females and 6 males) examined facial photographs of 14 patients. All 14 patients were Korean ( 7 males and 7 females) and underwent surgeryfirst orthognathic correction, facial bone contouring and followed by post-surgical orthodontic treatments by an oral surgeon, a plastic surgeon and an orthodontist respectively. Korea and US orthodontists were provided same patients' photographs except 1 male patient for US orthodontists. Two groups (US and Korea) of examiners estimated patients' age after observing frontal repose, frontal smile, 45 degrees and profile views of final (T2) and initial (T1) photographs of each patient in such order. We particularly used this order because 1) patient concern 'frontal beauty' rather than their lateral profiles; 2) viewing initial (T1) photographs with skeletal malocclusions may mislead the reviewers. A total of fourteen patients were rated by 20 examiners. When adequate power (1- $\beta$ error probability) for $80 \%$ was calculated using G-Power assuming $\alpha$ value of 0.05 , an outcome interaction was detected with sample size of 20 (examiners) to obtain critical F-value 4.41 and effect size of 0.172 . We expressed the age differences (T2T1) as if a continuous variable, scores. The scores have a unit of year. Thus, a score showing a negative number indicates that the postop face (face after surgery) looks younger than the initial face. The data, sets of collection of scores, were analyzed to test group differences. Sample measurements were sub-grouped based on examiner's practicing years (younger $v s$ older group) and nationality (US $v s$ Korea). Generalized Linear Mixed Model (SPSS v.24) was used because 1) our data includes repeated measurements, 2) the mixed model conveniently compensates random effects for categorical variable, and 3) the random effects were not assumed normal distributions, i.e. distribution free. All tests were non-distribution assumed, two-sided significance levels at $\mathrm{p}$ value less than 0.05 .

\section{Results}

Characteristics of participating orthodontists are summarized in table 1. Orthodontists (or examiners) in the US group are approximately 10 years older in average than Korea group although not significantly differ. Due to small numbers, we did not perform comparisons between rater subgroups based on gender. Figure 1 shows the differences in mean ages (T2 $\rightarrow \mathrm{T} 1)$ assessed by 10 Korean versus 10 US orthodontists.

\section{Comparisons between younger $v s$ older examiners}

Table 2 exhibits the results; a significant difference $(\mathrm{p}<0.05)$ was merged among Korean orthodontists when younger group was compared with older group. Korean Younger group estimated patient's age approximately 1 year younger than Korean Older group (1.84 for younger $v s 0.80$ for older). This result probably influenced the result from total group when Korea and US orthodontists were pooled. Estimation of postop faces by the Younger group (Korea+US) was 1.73

Table 1: Comparisons in years in practice between orthodontists in Korea vs US using Mann- Whitney $U$ test. The numbers in parentheses indicate standard deviations (SD).

\begin{tabular}{|l|c|c|c|c|c|}
\hline \multirow{2}{*}{} & \multicolumn{2}{|c|}{ Korea } & \multicolumn{2}{c|}{ US } & \\
\cline { 2 - 6 } & $\mathrm{N}$ & Years in practice & $\mathrm{N}$ & Years in practice & $\mathrm{P}$ \\
\hline Younger group & 4 & $6.8(2.87)$ & 3 & $3.3(2.52)$ & 0.163 \\
\hline Older group & 6 & $21.8(5.31)$ & 7 & $33.6(15.15)$ & 0.234 \\
\hline Total & 10 & $15.8(8.89)$ & 10 & $24.5(19.18)$ & 0.481 \\
\hline
\end{tabular}

Table 2: Comparisons between Younger vs Older groups on age differences (estimated Age at T1 - estimated Age at T2). Numbers with negative sign indicate reduced age perception in year $\mathrm{N}$ indicates the number of Scores on each patient by orthodontists. The numbers in parentheses indicate standard deviations (SD).

\begin{tabular}{|c|c|c|c|c|c|c|c|}
\hline & \multicolumn{3}{|c|}{ Younger group } & \multicolumn{3}{|c|}{ Older group } & \multirow[b]{2}{*}{$\mathbf{P}$} \\
\hline & $\mathbf{N}$ & Mean & (SD) & $\mathbf{N}$ & Mean & (SD) & \\
\hline \multicolumn{8}{|c|}{ All (males and females patients) } \\
\hline Korea & 56 & -1.84 & $(2.23)$ & 84 & -0.8 & $(2.65)$ & 0.013 \\
\hline US & 39 & -1.56 & $(4.42)$ & 91 & 0.2 & (5.47) & 0.044 \\
\hline Total (Korea+US) & 95 & -1.73 & $(3.29)$ & 175 & -0.28 & $(4.37)$ & $<0.001$ \\
\hline \multicolumn{8}{|c|}{ Female patients only } \\
\hline Korea & 28 & -1.61 & $(2.53)$ & 42 & -0.64 & $(2.55)$ & 0.095 \\
\hline US & 21 & -0.52 & $(5.01)$ & 49 & 1.98 & (6.24) & 0.084 \\
\hline Total (Korea+US) & 49 & -0.52 & $(3.79)$ & 91 & 0.77 & $(5.05)$ & 0.014 \\
\hline \multicolumn{8}{|l|}{ Male patients only } \\
\hline Korea & 28 & -2.07 & (1.9 & 42 & -0.95 & (2.76) & 0.066 \\
\hline US & 18 & -2.78 & (3.37) & 42 & -1.88 & (3.46) & 0.217 \\
\hline Total (Korea+US) & 46 & -2.35 & $(2.57)$ & 84 & -1.42 & (3.14) & 0.068 \\
\hline
\end{tabular}

compared to 0.28 by the Older group at the $\mathrm{p}<0.01$ level. This tendency was influenced by evaluations on female faces. When 7 female faces were examined by Korean and US examiners i.e., Total, Younger group estimated them 1.14 years younger compared older group who estimated postop faces of the females look 0.77 year older than initial photographs. This difference was significant at $\mathrm{p}<0.05$.

\section{Comparisons between Korean vs US examiners}

When compared table 3, the estimation on female faces by US group (Younger+Older) significantly differs from that of Korea group at $\mathrm{p}<0.01$ ( 1.03 younger by Korea Total $v s 1.23$ older by US Total). US orthodontists perceived that the female patients looked older after the operations. This significant disparity was led by US older group. The Korean older group estimates postop female faces would look 0.65 year younger than their initials. However, US older group estimated female postop faces appear 1.98 years older than initial photos. Yet, US younger group shares the same opinion with the Korean younger group (1.61 for Korea vs 0.52 for US).

\section{Discussion and Conclusion}

Unexpectedly, a significant generation-gap was found in both nationalities (US and Korea) despite no difference in their expertopinions was evident between the two nationalities. However, both older groups perceived less age difference between $\mathrm{T} 2$ and $\mathrm{T} 1$ than the younger groups in US and Korea. In comparison with male patients, the age estimation for female postop (T2) patients revealed a stronger intergenerational gap. In particular, the outcome the U.S. Older group produced was significantly different, i.e. T2 ages increased significantly after the surgeries (Figure 1). Here, we attempt to explain this distinctiveness of US Older group based on an old assertion, "Facial attraction is based on familiarity" [8,9]. Attractiveness ratings negatively correlate with distinctiveness ratings. They showed that attractiveness ratings were positively correlated with familiarity ratings. If their conclusion is correct, then the less familiarity for the US Older group to Asian female faces would have led them 

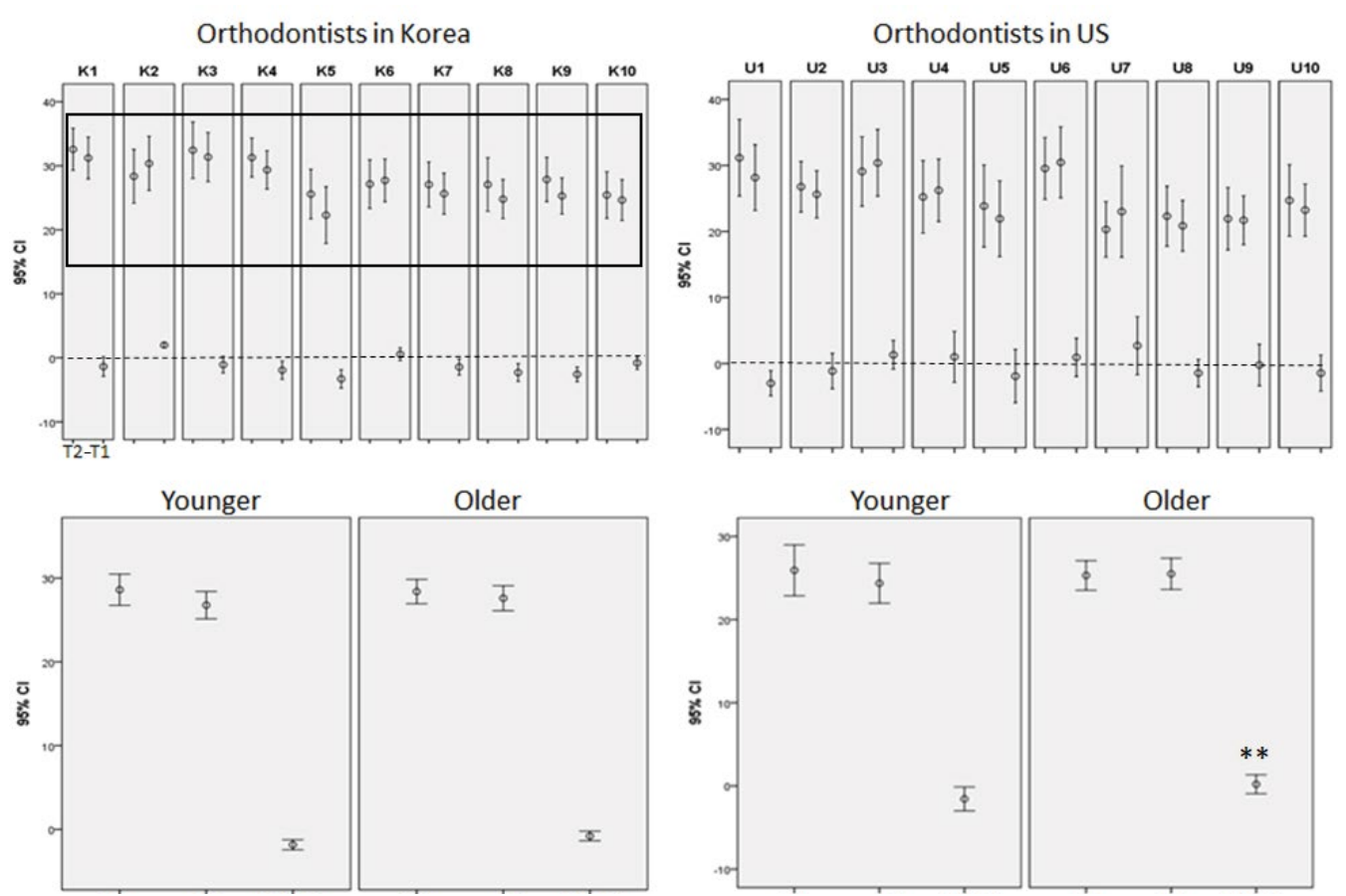

Figure 1: Differences in mean values ( $\mathrm{T} 1 \rightarrow \mathrm{T} 2)$ measured on 14 patients by 10 Korean and 10 US orthodontists. Average age of the patients measured by Orthodontist K2, K6, U3, U4, U6, and U7 (above dotted lines in the upper panels) increased at T2. Particularly, orthodontists belong to US older group perceived patients' face appear older at T2 (Note asterisk ${ }^{* *}$ ).

Table 3: Comparisons between orthodontists in Korea vs in US. The numbers in parentheses.

\begin{tabular}{|c|c|c|c|c|c|c|c|}
\hline & \multicolumn{3}{|c|}{ Korea $(\mathrm{N}=10)$} & \multicolumn{3}{|c|}{ US ( $N=10)$} & \multirow[b]{2}{*}{$\mathbf{P}$} \\
\hline & $\mathbf{N}$ & Mean & (SD) & $\mathbf{N}$ & Mean & (SD) & \\
\hline \multicolumn{8}{|c|}{ All (males and females patients) } \\
\hline Younger group & 56 & -1.84 & $(2.23)$ & 39 & -1.56 & $(4.42)$ & 0.626 \\
\hline Older group & 84 & -0.8 & $(2.65)$ & 91 & 0.2 & $(5.47)$ & 0.104 \\
\hline Total (Younger+Older) & 140 & -1.21 & $(2.53)$ & 130 & -0.33 & $(5.23)$ & 0.052 \\
\hline \multicolumn{8}{|l|}{ Female patients only } \\
\hline Younger group & 28 & -1.61 & $(2.53)$ & 21 & -0.52 & $(5.01)$ & 0.16 \\
\hline Older group & 42 & -0.64 & $(2.55)$ & 49 & 1.98 & $(6.24)$ & 0.01 \\
\hline Total (Younger+Older) & 70 & -1.03 & $(2.57)$ & 70 & 1.23 & $(5.97)$ & 0.002 \\
\hline \multicolumn{8}{|l|}{ Male patients only } \\
\hline Younger group & 28 & -2.07 & (1.9) & 18 & -2.78 & $(3.37)$ & 0.368 \\
\hline Older group & 42 & -0.95 & $(2.76)$ & 42 & -1.88 & $(3.46)$ & 0.183 \\
\hline Total (Younger+Older) & 70 & -1.4 & (2.5) & 60 & -2.15 & (3.43) & 0.16 \\
\hline
\end{tabular}

toward their evaluations. This may be one of plausible answers to their perceptions on the female T2 faces. Is "averageness hypothesis" proven to be true? The averageness hypothesis asserts an average face is attractive. A previous work [10] on facial shape and attractiveness conclude, however, that "highly attractive faces are systematically different in shape from average" in their comparison study between Caucasian and Japanese faces. More interestingly, regarding the direction' of preference on attractiveness, these two ethnic groups pointed the same direction [11]. This evidence indicates that we do not prefer an averageness, but "more than average" [12] which inferring a strong direction in the drive of perceptional changes in human facial beauty. Yet, orthodontic bracket-prescriptions mean to fix to average values over several decades. The current world we live is probably more spatially tighter and culturally open than ever $[7,13]$. Thus, intercultural or inter-social disparity may appear to be significantly reduced $[14,15]$. In this study, the average youthfulness one can achieve after the series of treatments including costly operations was less than 2 years. Then, one question raised as an orthodontist would be whether the procedures are beneficial to patients with respect to the risks and cost. Of course, 1-1.5 years for treatment time should be added to the 2 years of youthfulness each patient achieved in these samples for fair comparisons. For the answer, studies on quality of life for a long-term must follow. Another point to consider perhaps is that conventional orthodontic training focuses on profile evaluation of the face, yet less so on frontal beauty $[16,17]$. For better communication with patients, particularly with Asian patients with a broaden zygoma areas, more psychosocial research on frontal views is required in our orthodontic field. To be a good provider, we always mean to agree upon that "a doctor's up-to-date knowledge and skill provide the explicit scientific and often tacit experiential basis for such judgments" [18].

\section{Limitations}

What we measured may be occupational bias. Laypersons' opinion could be different; for instance, we could observe a significant sociocultural gap instead. Therefore, the next study should include more subgroups of raters based on more samples.

\section{References}

1. Faerber SJ, Carbon CC (2012) The power of liking: Highly sensitive aesthetic processing for guiding us through the world. Iperception 3: 553-561.

2. Park YS, Evans CA, Viana G, Anderson NK, Giddon DB (2006) Profile preferences of Korean American orthodontic patients and orthodontists. World J Orthod 7: 286-292. 
3. Evans CA (2000) Anteroposterior skeletal change: Growth modification. Semin Orthod 6: 21-32.

4. Yin L, Jiang M, Chen W, Smales RJ, Wang Q, et al. (2014) Differences in facial profile and dental esthetic perceptions between young adults and orthodontists. Am J Orthod Dentofacial Orthop 145: 750756.

5. Eslami N, Omidkhoda M, Shafaee H, Mozhdehifard M (2016) Comparison of esthetics perception and satisfaction of facial profile among male adolescents and adults with different profiles. J Orthod Sci 5: 47-51.

6. Toureno L, Kook YA, Bayome M, Park JH (2014) The effect of western adaptation of Hispanic-Americans on their assessment of Korean facial profiles. Korean J Orthod 44: 28-35.

7. Coetzee V, Greeff JM, Stephen ID, Perrett DI (2014) Cross-cultural agreement in facial attractiveness preferences: the role of ethnicity and gender. PLoS One 9: e99629.

8. Kiiski HS, Cullen B, Clavin SL, Newell FN (2016) Perceptual and Social Attributes Underlining Age-Related Preferences for Faces. Front Hum Neurosci 10: 437.

9. Peskin M, Newell FN (2004) Familiarity breeds attraction: effects of exposure on the attractiveness of typical and distinctive faces. Perception 33: 147-157.

10. Perrett DI, May KA, Yoshikawa S (1994) Facial shape and judgements of female attractiveness. Nature 368: 239-242.
11. Nguyen DD, Turley PK (1998) Changes in the Caucasian male facial profile as depicted in fashion magazines during the twentieth century. Am J Orthod Dentofacial Orthop 114: 208-217.

12. Hourfar J, Kinzinger GSM, Feifel H, Vehr VM, Lisson JA (2017) Effects of combined orthodontic-orthognathic treatment for class II and III correction on posterior airway space: Comparison of mono- and bignathic osteotomies. J Orofac Orthop 78: 455-465.

13. Bisson M, Grobbelaar A (2004) The esthetic properties of lips: a comparison of models and non models. Angle Orthod 74: 162-166.

14. Weeks DM, Thomas JR (2014) Beauty in a multicultural world. Facial Plast Surg Clin North Am 22: 337-341.

15. Foster EJ (1973) Profile preferences among diversified groups. Angle Orthod 43: 34-40.

16. Chen T, Khadka A, Hsu Y, Hu J, Wang D, et al. (2013) How to achieve a balanced and delicate lower third of the face in Orientals by mandibular contouring. J Plast Reconstr Aesthet Surg 66: 47-56.

17. Li Y, Hu Z, Ye B, Liu Y, Ren X, et al, (2016) Combined Use of Facial Osteoplasty and Orthognathic Surgery for Treatment of Dentofacial Deformities. J Oral Maxillofac Surg 74: e1- e12.

18. Working Party of the Royal College of Physicians (2005) Doctors in society. Medical professionalism in a changing world. Clin Med (Lond) 5: S5-S40. 